\title{
Evaluación oxidativa, microbiológica, sensorial y perfil de ácidos grasos de un yogur con ácido docosahexaenoico (DHA) extraído de aceite de microalgas
}

\author{
Oxidative, sensory and fatty acid profile evaluation of a yogurt with \\ docosahexaenoic acid (DHA) extracted from microalgae oil
}

\begin{abstract}
RESUMEN
Las necesidades de ácido docosahexaenoico (DHA), se incrementan en la mujer gestante para el desarrollo visual y neurológico del feto y el lactante. En este trabajo, se desarrolló y se evaluó un yogur adicionado con aceite de microalgas, que contribuyera a la recomendación dietaria de DHA en mujeres gestantes y lactantes. Se diseñaron tres formulaciones de yogur con 0,075; 0,125 y 0,175\% de aceite de microalga y se compararon con una muestra control. Se evaluaron propiedades fisicoquímicas, sensoriales, microbiológicas, perfil de ácidos grasos, potencial antioxidante (ABTS, fenoles totales) y peroxidación lipídica (sustancias reactivas al ácido tiobarbitúrico [TBARS]). Los yogures adicionados con el aceite de microalga cubrieron en 30, 45 y $63 \%$ las recomendaciones de DHA para mujeres gestantes y lactantes por porción (200 mL). Se observó estabilidad del ácido graso, excepto en la muestra de mayor adición del aceite. La muestra con adición de 0,125\% de aceite de microalga fue la de mejor calificación por el panel sensorial. Todas las muestras cumplieron con el estándar microbiológico y fisicoquímico para un yogur entero adicionado con dulce. Se observó potencial antioxidante promisorio en el yogur, capaz de proteger el DHA. Se concluye que las bebidas lácteas como el yogur son matrices adecuadas para la adición de aceite de microalga con la finalidad de aumentar el DHA en la dieta, especialmente en etapas en que las necesidades de este componente son más altas, como en periodo de gestación y lactancia.

Palabras clave: Ácido docosahexaenoico; Ácidos grasos n-3; Crypthecodinium cohnii; Gestación; Requerimientos; Yogur.
\end{abstract}

\footnotetext{
ABSTRACT

The needs for docosahexaenoic acid (DHA) are increased during pregnancy for the visual and neurological development of the fetus and the breastfed infant. In this study, a yogurt with microalgae oil added to contribute to the dietary recommendation of DHA in pregnant and breastfeeding women was developed and evaluated. Three yogurt formulations were designed with $0.075 ; 0.125$ and 0.175 microalgae oil percentage and compared with a control sample. Fatty acid profile, antioxidant potential (ABTS, total phenols),
}

\begin{abstract}
Briana Davahiva Gómez Ramírez ${ }^{1 *}$, José Uriel Sepúlveda Valencia², Andrés Felipe Alzate Arbelaez ${ }^{3}$, Jorge Mario Herrera², Benjamín Alberto Rojano ${ }^{3}$.
\end{abstract}

1.Escuela de Nutrición y Dietética, Universidad de Antioquia, Medellin- Colombia

2. Facultad de Ciencias Agrarias, Universidad Nacional de Colombia sede Medellín- Colombia. 3. Escuela de Química, Facultad de Ciencias, Universidad Nacional de Colombia sede Medellín-Colombia.

Dirigir correspondencia a: Briana Gómez, Escuela de Nutrición y Dietética, Grupo de investigación en Alimentación y Nutrición Humana, Universidad de Antioquia, Medellín-Colombia. Tel: +572199226 Email: briana.gomez@udea.edu.co

Este trabajo fue recibido el 06 de septiembre de 2019. Aceptado con modificaciones: 08 de enero de 2020. Aceptado para ser publicado: 12 de marzo de 2020.

lipid peroxidation (thiobarbitrical acid reactive substances [TBARS]), physicochemical, sensory, and microbiological properties were evaluated. Yogurts with microalgae oil added covered 30, 45 and $63 \%$ of DHA recommendations for pregnant and breastfeeding women per portion $(200 \mathrm{~mL})$. Fat acid stability was observed, except in the one with the greatest oil addition. The sample with $0.125 \%$ of microalgae oil added was rated the highest by the sensory panel. All samples met the microbiological and physicochemical standard for a whole yogurt added with sugar. Promising antioxidant potential capable of protecting DHA was observed in the yogurt. We conclude that dairy drinks such as yogurt are suitable matrices for adding microalgae oil in order to increase DHA in the diet, especially in stages 
where the needs of this component are higher as is the case during pregnancy and lactation periods.

Keywords: Crypthecodinium cohnii; Docosahexaenoic Acid; Gestation; Nutritional Requirements; n-3 Fatty Acids, Yoghurt stability.

\section{INTRODUCCIÓN}

Según la Organización Mundial de la Salud (OMS), durante la gestación y la lactancia se aumentan el riesgo de carencia de ácidos grasos poliinsaturados de cadena larga (AGPI-CL)' ${ }^{1}$, debido a que las reservas de los tejidos maternos suelen disminuir al utilizarse para el desarrollo del feto ${ }^{2,3}$. A los ácidos grasos que se les ha prestado más atención durante esta etapa son el ácido docosahexaenoico (DHA, C22:6n-3) y el ácido eicosapentaenoico (EPA, C20:5n-3), los cuales son derivados del metabolismo del ácido alfalinolénico (n-3), pues demuestran tener importantes efectos beneficiosos especialmente en el ámbito clínico, y con particular relevancia en el desarrollo y envejecimiento ${ }^{4}$. El DHA está involucrado en la fluidez de membrana, la formación de segundos mensajeros y la mejor producción de mediadores antiinflamatorios, a través de la modulación de la señalización $n^{5,6,7}$, dentro de estos mediadores se encuentra la regulación de los receptores activados por proliferadores peroxisomales (PPAR) ${ }^{8}$. Para cubrir las necesidades de los AGPI-CL, se recomienda el consumo de alimentos marinos, suplementos de aceites de pescado y de algas o leches maternizadas ${ }^{9}$; no obstante, el consumo de alimentos de mar está desfavorecido por el riesgo de contaminación con compuestos mercuriales que pueden atravesar la placenta ${ }^{10}$.

Los AGPI-CL son componentes importantes de la estructura de membranas, tienen un papel clave en su fluidez y en la homeostasis del calcio celular ${ }^{11}$. El DHA es abundante en las membranas de los fotorreceptores retinales y en el tejido neural, especialmente en la materia gris del cerebro, que comprende del 30 al 50\% de los lípidos de este tejido ${ }^{12,13,14}$ Es importante en el embarazo debido a que la tasa de desarrollo de retina y cerebro se acelera en el tercer trimestre de gestación y posteriormente durante la etapa de lactancia, debido a que continúa el desarrollo cerebral del infante y la acumulación de DHA, por lo menos durante los dos primeros años de vida ${ }^{15}$ Para ambos momentos, la mujer es quien va a proporcionar el DHA, ya sea por medio de la placenta, como ocurre en el periodo de gestación, o como componente natural de la leche materna durante el periodo de lactancia ${ }^{16}$. La OMS establece que se transfiere al feto una media de 14 mg/día de DHA durante las 40 semanas de gestación, siendo las 12 últimas semanas las de mayor transferencia, por el desarrollo acelerado del cerebro del feto ${ }^{17}$. De esta forma, si la madre recibe una alimentación con un aporte adecuado de AGPI, podrá aportar al feto (a través de los transportadores placentarios) y al recién nacido (a través de la leche) los requerimientos de AGPI-CL necesarios para el desarrollo normal del sistema nervioso y visual ${ }^{18}$. Bajo este panorama, es esencial la ingesta de DHA en mujeres gestantes y lactantes, ya sea de manera natural (consumo de alimentos) o por suplementación. En la actualidad, las recomendaciones de la ingesta para mujeres gestantes $y$ lactantes de EPA+DHA es de 288-313 mg/día, según la FDA (Food and Drug Administration, USA) y la OMS, de los cuáles $200 \mathrm{mg} /$ día corresponden a $\mathrm{DHA}^{17}$.

Por lo anterior, reconociendo las necesidades de las mujeres gestantes y lactantes y de la importancia de estos AGPI-CL en el desarrollo del feto, se han tratado de originar nuevas fuentes de DHA que sean seguras ${ }^{19}$. Esta exploración de fuentes alternativas de AGPI-CL ha logrado que se extraigan aceites de especies de microalgas, las cuales tienen el potencial de acumular lípidos en altas cantidades y tienen elevados contenidos de AGPI-CL, específicamente de DHA (40 y $50 \%$ del aceite extraído) ${ }^{20}$.

Se ha analizado el aceite extraído de microalgas respecto a su biodisponibilidad, toxicidad subcrónica, presencia de metales pesados y potencial mutagénico, declarándolo, hasta el momento, según la FDA, como un aceite seguro y biodisponible (que el cuerpo humano puede absorber $y$ utilizar), para ser adicionado a otros alimentos ${ }^{21}$. Sumado a lo anterior, su producción se basa en cultivos sostenibles, respecto a lo ambiental, lo que no sucede con la extracción de aceites de pescados u otras fuentes de ácidos grasos $\mathrm{n}-3^{22}$. Adicionalmente, el aceite resultante queda con excelentes características organolépticas, por su bajo olor y sabor residual ${ }^{23}$.

Para una adecuada absorción de estos ácidos grasos, se requiere de matrices alimentarias con contenido lipídico que vehiculicen adecuadamente los $\mathrm{AGPI}^{24}$. Los lácteos, en este sentido, se han convertido en alimentos comúnmente utilizados, que aseguran que los lípidos adicionados conserven su isomería, debido a que no están sometidos a altas temperaturas. El objetivo de este trabajo fue desarrollar y evaluar tres formulaciones de un yogur suplementado con diferentes cantidades de DHA por incorporación de aceite de microalgas.

\section{MATERIALES Y MÉTODOS}

El diseño es experimental y comparativo, se evaluó la adición de distintas cantidades de aceite de microalga (Crypthecodinium cohnii) en un producto lácteo fermentado tipo yogur.

\section{Formulación del producto}

Preparación del yogur: la leche entera de vaca fresca se calentó a $45^{\circ} \mathrm{C}$ a 1500 psi para su homogenización. Se pasterizó a $85^{\circ} \mathrm{C}$ por 15 minutos, cuando estuvo en $41,5^{\circ} \mathrm{C}$ se inoculó con una mezcla de Streptococcus thermophilus, Lactobacillus delbrueckii subsp. lactis y Lactobacillus delbrueckii subsp. bulgaricus, incubando hasta un $\mathrm{pH}=0,55$. Posteriormente, se adicionó $15 \%$ de la mezcla de pulpa de tomate de árbol (Solanum betaceum) con azúcar refinada en una proporción 1:1 (pulpa: azúcar), que previamente se pasterizó a $85{ }^{\circ} \mathrm{C}$ por 15 minutos. Una vez preparado el yogur, se adicionó el aceite de microalga conformando cuatro 
grupos experimentales, así: M0 o grupo control (sin aceite de microalga), M1, M2 y M3, con 0,075; 0,125 y 0,175\%, de aceite de microalga respectivamente. Esto proporciona 60,100 y $140 \mathrm{mg}$ de DHA por $200 \mathrm{~mL}$; y cubre el 30, 50 y $70 \%$ de la recomendación de DHA respectivamente para mujer gestante y lactante. Se realizaron dos lotes de cada muestra en tiempos diferentes. Cada lote fue almacenado a $4{ }^{\circ} \mathrm{C}$ para los análisis correspondientes.

\section{Aceite de microalga}

El aceite comercial utilizado fue extraído de microalgas de la especie Crypthecodinium cohnii (DHASCO ${ }^{\circledR}$, Martek Biosciences Corporation, Columbia, MD, USA). La composición de ácidos grasos del aceite se presenta en la tabla 1, en la que se observa una proporción de DHA superior al 40\% y del 2,5\% para EPA ${ }^{25}$. El aceite fue estabilizado con $\alpha$-tocoferol $(0,025 \%)$ y palmitato de ascorbilo (0,025\%).

\section{Cuantificación de ácidos grasos}

Para la extracción de la grasa en yogur, se siguió el método de Roese-Gottlieb ${ }^{26}$; se pesaron 10 g de muestra en un tubo de vidrio, $100 \mathrm{mg}$ de butilhidroxitolueno (BHT) y se agregó $2 \mathrm{~mL}$ del estándar interno gliceril triundecanoato (5 $\mathrm{mg} / \mathrm{mL}$ ). Luego se adicionó 1,25 mL de amoniaco al 25\%, se agitó por $1 \mathrm{~min}$, se adicionó $10 \mathrm{~mL}$ de etanol y se agitó de nuevo por 1 min para la separación y precipitación de la proteína. Para la extracción de la grasa, se adicionó 15 $\mathrm{mL}$ de dietil éter, se agitó por 1 minuto, y luego, $15 \mathrm{~mL}$ de éter de petróleo y se agitó de nuevo por 1 minuto, se permitió la separación de las fases. La fase etérea se trasvasó a un Erlenmeyer de $50 \mathrm{~mL}$, se repitió la extracción para la fase restante, pero esta vez con una adición de $5 \mathrm{~mL}$. Posteriormente, se evaporó la mezcla de éteres y se pesó el residuo graso. Para la conversión del extracto lipídico a ésteres metílicos, se siguió el método AOAC 969.33727; al extracto seco se le adicionó $5 \mathrm{~mL}$ de $\mathrm{NaOH} / \mathrm{MeOH}$ 0,5 N y se dejó en reflujo hasta la desaparición de los glóbulos de grasa, luego se adicionó $5 \mathrm{~mL}$ de $\mathrm{BF}_{3} / \mathrm{MeOH}$ al $12 \%$ y se dejó en reflujo por 2 min más y, por último, se adicionó 4 $\mathrm{mL}$ de hexano en reflujo por 1 min más. Se retiró del calor y se adicionó $15 \mathrm{~mL}$ de una solución saturada de $\mathrm{NaCl}$, se tomó la capa superior (fase orgánica) y se llevó a un tubo con sulfato de sodio anhidro. Para la determinación de los ácidos grasos, se usó un cromatógrafo de gases Agilent ${ }^{\circledR}$ 7890B con detector FID, automuestreador 7963A, inyección split 100:1, columna capilar de sílica altamente polar TRCN$100(60 \mathrm{~m} \times 0,25 \mathrm{~mm}$ d.i. x 0,20 $\mu \mathrm{m})$ marca Teknokroma, Barcelona - España. El volumen de inyección fue de $1 \mu \mathrm{L}$ y el programa de temperatura del horno fue inicialmente a $100{ }^{\circ} \mathrm{C}$, con incrementos de $8{ }^{\circ} \mathrm{C} / \mathrm{min}$ hasta $145^{\circ} \mathrm{C}$ por 5 min y luego a un incremento de $2^{\circ} \mathrm{C} / \mathrm{min}$ hasta $220^{\circ} \mathrm{C}$ por

Tabla 1. Composición lipídica del aceite $\mathrm{DHASCO}{ }^{\circledR}$, usado en la formulación de los yogures.

\begin{tabular}{lll|} 
Ácido graso & Nombre común & $\begin{array}{c}\text { Nivel de ácidos grasos } \\
\text { (\%) del total de ácidos grasos) } \\
\text { Aceite DHASCO }^{\circledR}\end{array}$ \\
\hline C10:0 & Ácido cáprico & 0,6 \\
C12:0 & Ácido láurico & 4,5 \\
C14:0 & Ácido miristico & 15,4 \\
C14:1 n-5 & Ácido miristoleico & 0,2 \\
C16:0 & Ácido palmítico & 11,6 \\
C16:1 n-7 & Ácido palmitoleico & 2,3 \\
C18:0 & Ácido esteárico & 0,3 \\
C18:1 n9 & Ácido oleico & 11,3 \\
C18:2 n-6 & Ácido linoleico (LA) & 0,8 \\
C18:3 n3 & Ácido linolenico (LNA) & 0,2 \\
C20:0 & Ácido araquidico & 0,1 \\
C20:1n9 & Ácido eicosenoico & 0,1 \\
C22:0 & Ácido behenico & 0,1 \\
C22:6 n-3 & Ácido docosahexaenoico (DHA) & 51,7 \\
\hline
\end{tabular}

Fuente: Arterburn (25). 
1 min. Gas helio se usó como gas de arrastre y las señales se recogieron y procesaron mediante el software OpenLab CDS ChemStation. La identificación de los ácidos grasos se hizo por comparación con los tiempos de retención en una mezcla de solución estándar y la cuantificación fue realizada de acuerdo con el método oficial AOAC $996.06^{28}$. Los resultados fueron expresados en g de ácido graso por $100 \mathrm{~g}$ de yogur.

\section{Análisis microbiológicos}

Las pruebas microbiológicas se realizaron en los días 2 y 15 de almacenamiento en refrigeración a $4{ }^{\circ} \mathrm{C}$; fueron determinados los marcadores de contaminación microbiológica: coliformes totales y fecales (FDA-BAM/NMP) ${ }^{29}$, hongos y levaduras con recuento en placa (ISO 6611) ${ }^{30}$. Se siguieron las características microbiológicas para yogur descritas según normatividad ${ }^{31}$.

\section{Análisis sensorial}

Posterior a la conformidad de los análisis microbiológicos, las muestras se llevaron al Laboratorio de Análisis Sensorial. Dieciocho jueces entrenados realizaron pruebas de ordenación en calidad general para las muestras de acuerdo con la metodología señalada por las Normas Técnicas ${ }^{32}$. Se realizó prueba multidimensional ${ }^{33,34}$ para la muestra de mejor calidad sensorial, en esta se identificó y seleccionó el conjunto de descriptores relevantes, valorando las intensidades con jueces entrenados en una escala de calificación de 0 a 5 para todos los descriptores. Excepto para la calidad general, en esta se utilizó una escala de 1 a 3, donde 3 es alto y 1 es bajo.

\section{Análisis fisicoquímico}

Se determinó la acidez por titulación con $\mathrm{NaOH} 0,1 \mathrm{~N}$, utilizando como indicador fenolftaleína (AOAC 942,15) ${ }^{35}$. Se midió humedad en deshidratador hasta peso constante (GTC 1,14) y materia grasa según lo descrito en el análisis de ácidos grasos y proteínas por el método de micro Kjeldhal $\left(\right.$ AOAC 954,01) ${ }^{36}$.

\section{Determinación de fenoles totales}

Se realizó utilizando el método colorimétrico de FolinCiocalteu modificado por Sánchez et $\mathrm{al}^{37}$. En un tubo de reacción se adicionó $50 \mu \mathrm{L}$ de yogur, $425 \mu \mathrm{L}$ de agua destilada y $125 \mu \mathrm{L}$ del reactivo Folin-Ciocalteu. Se agitó y luego se dejó en reposo por 6 minutos. Posteriormente, se adicionó $400 \mu \mathrm{L}$ de $\mathrm{Na}_{2} \mathrm{CO}_{3}$ al $7,1 \%$. Después de $1 \mathrm{~h}$ en la oscuridad, se leyó la absorbancia a $760 \mathrm{~nm}$. Se construyó una curva patrón usando como estándar ácido gálico. Los análisis se realizaron por triplicado y los resultados se expresaron como mg de ácido gálico/L de muestra. Se realizó triplicado por medición cada 6 días y se promediaron los tres resultados disponibles.

\section{Método del radical catiónico ABTS•+}

Se determinó la capacidad para atrapar radicales libres empleando el método del radical catiónico ABTS
[2,2'-Azino-bis (3-etilbenzotiazolina-6-ácido sulfónico) sal diamonio. Se mezclaron en tubos de ensayo $10 \mu \mathrm{L}$ de muestra y $990 \mu \mathrm{L}$ de solución de ABTS, por separado se preparó una referencia y un blanco, en el caso de la referencia se utilizaron $10 \mu \mathrm{L}$ de agua destilada más $990 \mu \mathrm{L}$ de ABTS. La absorbancia fue registrada a $734 \mathrm{~nm}$ después de 30 min de reacción a temperatura ambiente. El cálculo de la actividad antioxidante se realizó empleando una curva de calibración y usando Trolox como antioxidante de referencia (Sigma-Aldrich, Misuri, Estados Unidos), los resultados fueron reportados como TEAC: Trolox Equivalent Antioxidant Capacity ( $\mu \mathrm{mol}$ de Trolox/L de muestra). Se realizó triplicado por medición cada 6 días y se promediaron los tres resultados disponibles ${ }^{37}$.

\section{Estabilidad oxidativa \\ Sustancias reactivas al ácido tiobarbitúrico (TBARS)}

Se realizó según la metodología descrita por Sánchez et $\mathrm{a}^{38}$. Los reactivos de TBA (ácido 2 -tiobarbitúrico 0,67\%), TCA (ácido tricloroacético 15\%) y la muestra fueron mezclados, agitados y llevados a un baño de agua a $90{ }^{\circ} \mathrm{C}$. Luego de enfriarla, se adicionó $600 \mu \mathrm{L}$ de butanol. El complejo fluorescente formado fue leído en un espectrofluorímetro (Perkin Elmer LS55, Boston, MA, Estados Unidos) a una $\lambda$ de excitación de $500 \mathrm{~nm}$ y $\lambda$ de emisión $550 \mathrm{~nm}$. Los resultados fueron expresados como equivalentes de malondialdehído (MDA) (nmol/g de muestra). Se realizó triplicado por medición cada 6 días y se promediaron los tres resultados disponibles.

\section{Aspectos éticos}

Las muestras de yogur antes de ser evaluadas por los panelistas entrenados cumplieron con los análisis microbiológicos. Constituyéndose en una investigación de bajo riesgo. Los jueces entrenados reciben bonificación con los estudios técnicos realizados en el Laboratorio de análisis sensorial de alimentos.

\section{Análisis estadístico}

Se efectuó análisis estadístico a partir de medidas descriptivas como la media, mediana, frecuencias y desviación estándar. Los valores para el perfil de ácidos grasos, microbiológico y de propiedades fisicoquímicas están expresados en el promedio de los dos lotes de yogur realizados. Se aplicó el Test de Shapiro-Wilk para análisis de exploración en la prueba sensorial y para análisis antioxidante. En el análisis sensorial se aplicó la prueba de Friedman, para determinar significancia. Se realizó un ANOVA de medidas repetidas o Wilcoxon según los supuestos de normalidad, para el análisis de antioxidantes y peroxidación; cuando estas diferencias fueron significativas, se ejecutó prueba post hoc de ajuste de Bonferroni para las comparaciones múltiples. Para el procesamiento de datos en el análisis fisicoquímico, microbiológico y perfil de ácidos grasos, se utilizó la aplicación Microsoft Excel. Para el análisis estadístico del potencial antioxidante y del 
análisis sensorial se empleó el paquete estadístico de SPSS versión 19. El nivel de significancia fue de 0,05.

\section{RESULTADOS}

Análisis fisicoquímico: en la tabla 2 se presentan los resultados de los análisis fisicoquímicos de las muestras de yogur. Todas las formulaciones cumplen con las características de yogur entero con dulce, el cual plantea un mínimo de materia grasa de 2,5\% ${ }^{31}$. En las muestras M1, M2 y M3, se observan valores más altos de grasa por la adición del aceite de microalga. El contenido de proteína no presenta diferencias significativas entre las muestras. Los sólidos totales muestran tendencia al aumento por la incorporación del aceite, pero sin presentar diferencias significativas.

Análisis microbiológico: los análisis microbiológicos se muestran en la tabla 3. Se observa que todas las muestras cumplen parámetros de buena calidad microbiológica según la normatividad. La única muestra que se observa con un nivel superior al permitido en levaduras es la M3, en el día 15 de almacenamiento; sin embargo, se puede catalogar como aceptable en calidad microbiológica, debido a que la normatividad establece su máximo para este indicador en 500 UFC.

Análisis sensorial: en la prueba de Friedman se evidenció que existen diferencias significativas entre $M 0$ y M3, M1 y $M 3, M 2$ y $M 3(p \leq 0,05)$ en calidad general, sin embrago, no existe diferencia entre la muestra $\mathrm{M} 0$ y M1, M0 y M2 y M1 y M2. El atributo de calidad general fue mayor para la muestra M2, luego M1 y M3. Las características destacadas por los jueces para que la muestra M2 tuviera la mayor aceptabilidad fueron la presencia de mayor cuerpo en boca, mayor viscosidad, sabor frutal y percepción de balance en sabor. El perfil sensorial completo para la muestra M2 se muestra en la figura 1.

Tabla 2. Características fisicoquímicas del yogur con adición de aceite de microalga a $4{ }^{\circ} \mathrm{C}$, en el día 5 de almacenamiento.

\begin{tabular}{|c|c|c|c|c|c|}
\hline \multirow{2}{*}{ Análisis fisicoquímico } & \multicolumn{5}{|c|}{ Promedio \pm desviación estándar } \\
\hline & Mo & M1 & M2 & M3 & Valor de $\mathrm{p}^{*}$ \\
\hline Humedad (g/100 g) & $80,6 \pm 1,4$ & $80,7 \pm 1,1$ & $80,6 \pm 1,3$ & $80,6 \pm 1,2$ & 0,999 \\
\hline Sólidos totales $(\mathrm{g} / 100 \mathrm{~g})$ & $19,4 \pm 1,4$ & $19,4 \pm 1,2$ & $19,4 \pm 1,3$ & $19,4 \pm 1,2$ & 0,999 \\
\hline Grasa total (g/100 g) & $2,5 \pm 0,2$ & $2,6 \pm 0,2$ & $2,6 \pm 0,2$ & $2,7 \pm 0,2$ & 0,103 \\
\hline Proteína total $(\mathrm{g} / 100 \mathrm{~g})$ & $2,2 \pm 0,2$ & $2,3 \pm 0,2$ & $2,3 \pm 0,2$ & $2,3 \pm 0,4$ & 0,936 \\
\hline
\end{tabular}

*ANOVA.

Tabla 3. Recuento de coliformes totales y fecales y mohos y levaduras en las muestras de yogur con adición de aceite de microalga almacenado a $4{ }^{\circ} \mathrm{C}$.

\begin{tabular}{|c|c|c|c|c|c|}
\hline \multicolumn{3}{|c|}{$\begin{array}{l}\text { Análisis } \\
\text { microbiológico }\end{array}$} & \multirow{2}{*}{$\begin{array}{c}\text { NMP } \\
\text { coliformes/g o } \mathbf{~ m L} \\
3,0\end{array}$} & \multirow{2}{*}{$\begin{array}{c}\text { NMP } \\
\text { coliformes fecales/g o mL } \\
<3\end{array}$} & \multirow{2}{*}{$\begin{array}{c}\begin{array}{c}\text { Recuento mohos y } \\
\text { levaduras UFC/g o mL }\end{array} \\
\qquad 100 \text { UFC }\end{array}$} \\
\hline MO & Día & 2 & & & \\
\hline & Día & 15 & $<3$ & $<3$ & 10 UFC tipo mohos \\
\hline \multirow[t]{2}{*}{ M1 } & Día & 2 & 9,1 & $<3$ & $<100$ UFC \\
\hline & Día & 15 & $<3$ & $<3$ & 10 UFC tipo mohos \\
\hline \multirow[t]{2}{*}{ M2 } & Día & 2 & 3,6 & $<3$ & $<100$ UFC \\
\hline & Día & 15 & $<3$ & $<3$ & 170 UFC tipo levaduras \\
\hline \multirow[t]{2}{*}{ M3 } & Día & 2 & 75,0 & $<3$ & $<100$ UFC \\
\hline & Día & 15 & 3,6 & $<3$ & 220 UFC tipo levaduras \\
\hline
\end{tabular}

NMP: número más probable. UFC: unidades formadoras de colonias. 


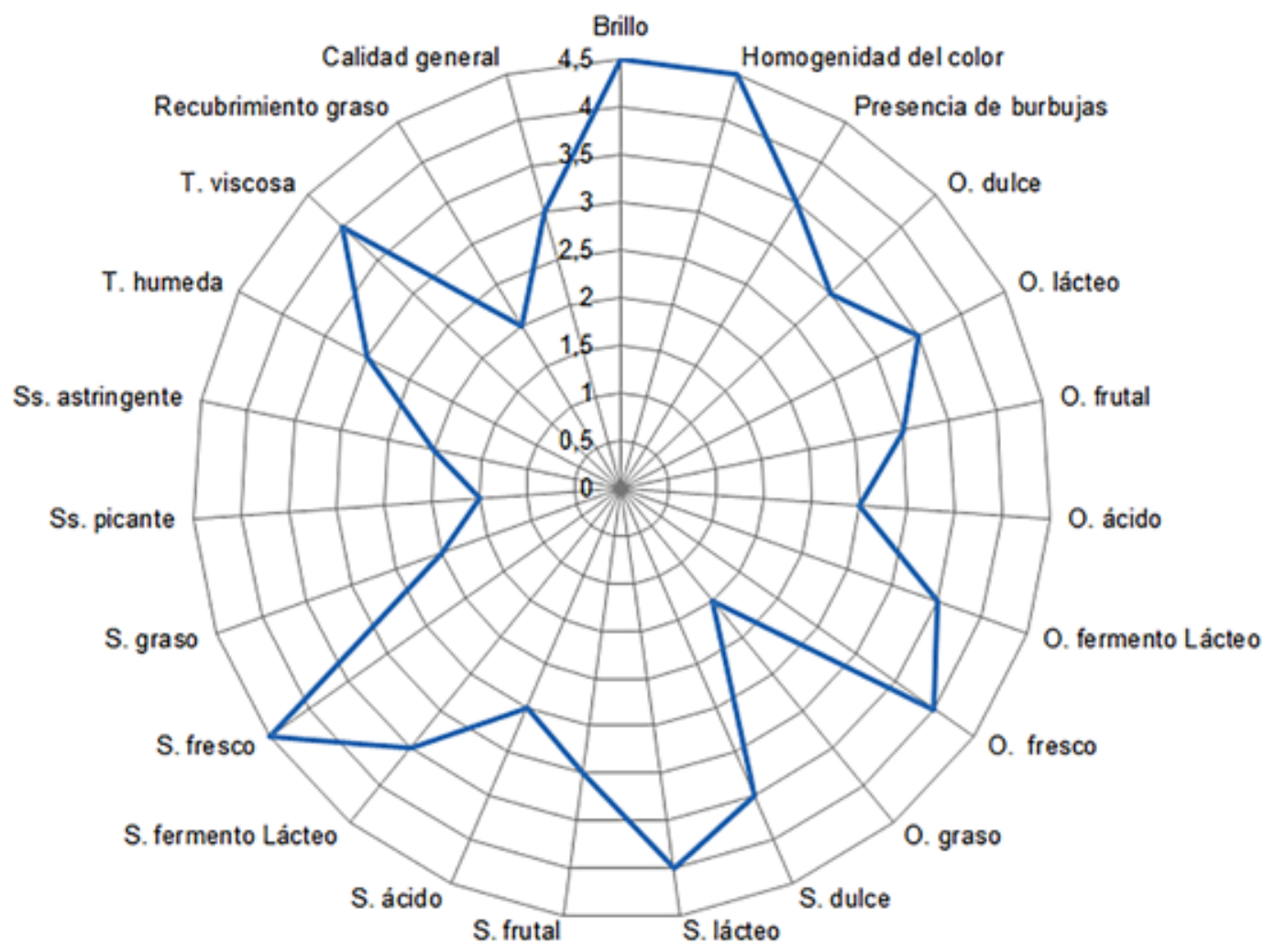

Figura 1: Perfil sensorial por aproximación multidimensional para M2, muestra que presentó la mayor calidad general según prueba de ordenación en el análisis sensorial a $4{ }^{\circ} \mathrm{C}$.

O: Olor; S: sabor; T: textura; Ss: sensación.

Perfil de ácidos grasos: el perfil de ácidos grasos de las muestras de yogures se muestra en la tabla 4, para el día 2 y 15 de almacenamiento. Se identifica que la adición del aceite de microalga modifica especialmente el DHA (C22:6n3), a su vez, esto afecta el contenido total de AGPI de las muestras que tienen la incorporación del aceite. Se destacan en todas las muestras de yogures el ácido palmítico (C16:0), en cantidades entre 0,68 y 0,72 g/100 g, y el ácido mirístico (C14:0) (0,20 a 0,24 g/100 g) como ácidos grasos saturados y oleicos (C18:1n9c), y como monoinsaturados en cantidades de 0,64 a 0,69 g/100 g, los cuales están naturalmente en productos lácteos.

En la muestra M1 se identificó 0,03 g/100 g DHA, siendo esta la cantidad que se esperaba determinar por el aceite de microalga adicionado, en la M2 se detectó $0,04 \mathrm{~g} / 100$ $\mathrm{g}$, siendo cercano a la adición de DHA que se esperaba $(0,05 \mathrm{~g} / 100 \mathrm{~g})$, y en M3 con contenido de DHA de 0,06 g/100 g es inferior a la adición programada (0,07 g/100 g). No se observaron variaciones en el DHA entre el día 2 y 15 para la muestra M1 y M2; no obstante, para la muestra M3 se observa una reducción en el día 15 de 0,02 g/100 g.

Al analizar la cantidad identificada del DHA en el perfil de ácidos grasos, se puede determinar que, en una porción de $200 \mathrm{~mL}$ de cada uno de los yogures, las muestras M1, M2 y M3 estarían aportando, en su respectivo orden, 60, 90 y $126 \mathrm{mg} / 200 \mathrm{~mL}$ de yogur, lo que corresponde al 30, 45 y $63 \%$ de la recomendación de DHA para la mujer gestante y lactante por porción de producto. La relación n-6/n-3 disminuye a medida que aumenta la adición del aceite en cada una de las muestras. La muestra control (M0) tiene una relación de aproximadamente 3,3, y en las demás la relación disminuye a aproximadamente 0,$8 ; 0,6$ y 0,4 , respectivamente, para $M 1, M 2$ y $M 3$. 
Tabla 4. Perfil de ácidos grasos en las muestras de yogur con adición de aceite de microalga a $4{ }^{\circ} \mathrm{C}$, en el día 2 y 15 de almacenamiento.

\begin{tabular}{|c|c|c|c|c|c|c|c|c|}
\hline & \multicolumn{8}{|c|}{ Promedio } \\
\hline & \multicolumn{2}{|c|}{ MO } & \multicolumn{2}{|c|}{ M1 } & \multicolumn{2}{|c|}{ M2 } & \multicolumn{2}{|c|}{ M3 } \\
\hline & $\begin{array}{c}\text { Día } 2 \\
\text { g/100 g }\end{array}$ & $\begin{array}{l}\text { Día } 15 \\
\text { g/100 g }\end{array}$ & $\begin{array}{c}\text { Día } 2 \\
\text { g/100 g }\end{array}$ & $\begin{array}{l}\text { Día } 15 \\
\text { g/100 g }\end{array}$ & $\begin{array}{c}\text { Día } 2 \\
\text { g/100 g }\end{array}$ & $\begin{array}{l}\text { Día } 15 \\
\text { g/100 g }\end{array}$ & $\begin{array}{c}\text { Día } 2 \\
\text { g/100 g }\end{array}$ & $\begin{array}{l}\text { Día } 15 \\
\text { g/100 g }\end{array}$ \\
\hline Ácido butírico (C4:0) & 0,06 & 0,04 & 0,04 & 0,05 & 0,04 & 0,05 & 0,04 & 0,05 \\
\hline Ácido caproico (C6:0) & 0,03 & 0,03 & 0,03 & 0,03 & 0,03 & 0,03 & 0,03 & 0,03 \\
\hline Ácido caprílico (C8:0) & 0,02 & 0,02 & 0,02 & 0,02 & 0,02 & 0,02 & 0,02 & 0,02 \\
\hline Ácido cáprico (C10:0) & 0,09 & 0,08 & 0,10 & 0,11 & 0,11 & 0,11 & 0,10 & 0,11 \\
\hline Ácido láurico (C12:0) & 0,06 & 0,06 & 0,07 & 0,07 & 0,07 & 0,07 & 0,07 & 0,07 \\
\hline Ácido mirístico (C14:0) & 0,23 & 0,24 & 0,24 & 0,25 & 0,24 & 0,20 & 0,24 & 0,25 \\
\hline Ácido palmítico (C16:0) & 0,70 & 0,70 & 0,69 & 0,71 & 0,68 & 0,72 & 0,69 & 0,71 \\
\hline Ácido esteárico (C18:0) & 0,37 & 0,37 & 0,36 & 0,37 & 0,35 & 0,38 & 0,36 & 0,37 \\
\hline Ácido oleico (C18:1n9c) & 0,65 & 0,65 & 0,66 & 0,68 & 0,65 & 0,69 & 0,66 & 0,68 \\
\hline Ácido linoleico (C18:2n6c) & 0,03 & 0,03 & 0,03 & 0,03 & 0,03 & 0,03 & 0,03 & 0,03 \\
\hline Ácido a-linolénico (C18:3n3) & 0,01 & 0,01 & 0,01 & 0,01 & 0,01 & 0,01 & 0,01 & 0,01 \\
\hline $\begin{array}{l}\text { Ácido cis-4,7,10,13,16,19- } \\
\text { docosahexaenoico (C22:6n3) }\end{array}$ & ND & ND & 0,03 & 0,03 & 0,04 & 0,04 & 0,06 & 0,04 \\
\hline Total saturada (\%) & 1,61 & 1,61 & 1,61 & 1,67 & 1,58 & 1,64 & 1,60 & 1,67 \\
\hline Total monoinsaturada (\%) & 0,70 & 0,70 & 0,71 & 0,74 & 0,70 & 0,75 & 0,72 & 0,73 \\
\hline Total poliinsaturada $(\%)$ & 0,04 & 0,05 & 0,08 & 0,08 & 0,09 & 0,09 & 0,11 & 0,09 \\
\hline
\end{tabular}

$\mathrm{ND}=$ no detectado.

Se omiten desviaciones estándar debido a que las milésimas era muy pequeñas.

\section{Actividad antioxidante}

Radical catiónico ABTS: la figura 2-A muestra el comportamiento del radical catiónico ABTS a lo largo de 34 días de almacenamiento de las muestras de yogur. Se puede observar gran variabilidad de los datos detectando cómo se mantienen los valores de ABTS a pesar del paso de los días. En la muestra M0, para el día 1, se identificó el radical catiónico ABTS 11791,1 $\mu \mathrm{mol}$ Trolox/L de muestra, el cual disminuye a 8060,8 $\mu \mathrm{mol}$ Trolox/L muestra en el día 13; posteriormente, se observa una tendencia al incremento hasta el día 27, con valores de 16837,5 $\mu \mathrm{mol}$ Trolox/L muestra. Esta misma tendencia de datos se observa para todas las muestras, con diferencias significativas en las mediciones a través de los días $(<0,05)$. Al realizar las comparaciones múltiples, se determina que la muestra M0 no tuvo diferencias significativas en los valores de ABTS con las muestras M2 y M3 $(>0,05)$.
Fenoles totales: los resultados de los fenoles totales durante el almacenamiento se observan en la figura 2-B. La muestra M0 presenta mayor contenido de fenoles totales en el transcurso de los días (entre 448,9 a 709,2 mg ácido gálico/L de muestra), en comparación con las muestras con adición de aceite. Se observa en todas las muestras que el contenido de fenoles totales tiene una tendencia a la diminución hasta el día $34(<0,05)$. Al realizar las comparaciones múltiples entre las muestras, se determina que no tuvieron diferencias significativas en el valor de fenoles totales, M1 con M2 y M2 con M3 $(>0,05)$.

\section{Estabilidad oxidativa}

Sustancias reactivas al ácido tiobarbitúrico (TBARS): se observa aumento en los valores de TBARS hidrofílico para el día 34 de almacenamiento (Figura 2-C); se identificaron en este punto la peroxidación lipídica de los AGPI. Lo 

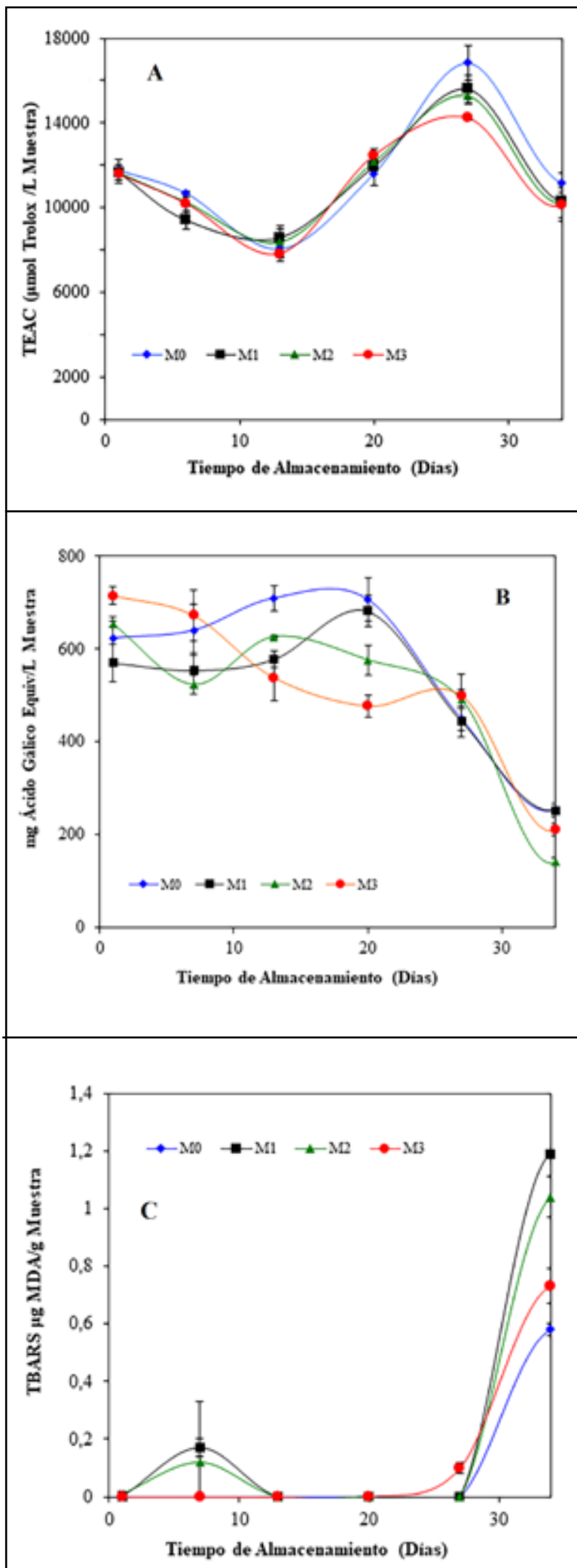

anterior coincide con la disminución de fenoles totales. La muestra M0 tiene los menores valores de TBARS, en comparación con las muestras con adición de aceite, le siguen las muestras M3, M2 y M1, que mostraron valores más altos (1,2 $\mu \mathrm{g}$ MDA/g muestra), pese a ello, al realizar las comparaciones múltiples entre las muestras se determina que no hay diferencias significativas en TBARS entre las muestras $(>0,05)$.

\section{DISCUSIÓN}

Los resultados de esta investigación indican que la adición de aceite de microalga con alto contenido de DHA (C22:6n3) a un yogur es una alternativa viable para cubrir parte de las recomendaciónes dietarias de DHA en mujeres gestantes y lactantes. Todas las muestras cumplieron con los parámetros microbiológicos y fisicoquímicos de un yogur entero con azúcar según la normatividad para este tipo de alimento ${ }^{31}$; adicionalmente, la adición y estabilidad del DHA fueron satisfactorias. El potencial antioxidante identificado en las diferentes formulaciones puede ayudar a proteger el ácido graso adicionado. Se identifica que el yogur con mejor evaluación general, determinada por el análisis sensorial de jueces entrenados, fue la que tuvo adición intermedia del aceite $(0,125 \%)$ (Figura 1$)$ y cubrió el $45 \%$ de la recomendación de DHA por porción de $200 \mathrm{~mL}$ (90 mg de DHA/200 mL de yogur) para el grupo poblacional en mención.

En este estudio se observan ventajas en la adición del aceite de microalga, en comparación con otras fuentes de ácidos grasos n-3. Por lo tanto, la adición de este aceite no tuvo problemas sensoriales en cuanto al olor o separación de fases en las formulaciones, a lo que se le suma la adecuada incorporación en la matriz láctea, debido a que en el análisis de ácidos grasos este aceite no cambia el perfil lipídico natural del yogur, solo incrementa el contenido de DHA, que es el ácido graso de interés en este trabajo (Tabla 4). Lo anterior es uno de los hechos más importantes, porque cuando se realiza la incorporación de AGPI n-3 de origen vegetal, estos ácidos pueden estar limitados en el acceso, por no poseer los metabolitos de mayor funcionalidad en salud como el EPA y el DHA. El pescado y el aceite de pescado son las fuentes comunes de AGPI-CL, pero se evidencian problemas de seguridad a causa de la posible acumulación de tóxicos en los peces. A su vez, la adición de aceite de pescado es limitada, debido a problemas asociados con su olor característico, sabor poco aceptable, baja estabilidad oxidativa y problemas en la sostenibilidad de la vida marina ${ }^{39}$. Por lo anterior, las microalgas han cobrado interés en los

Figura 2: Cambios durante el almacenamiento en la actividad antioxidante, contenido de polifenoles y peroxidación lipídica en diferentes formulaciones de yogur a $4{ }^{\circ} \mathrm{C}$. Panel A: ABTS ( $\mu \mathrm{M}$ Trolox/L de muestra). Panel B: polifenoles totales (mg de ácido gálico Eq./L de muestra). Panel C: TBARS ( $\mu g$ MDA/g de muestra). 
últimos años, pues ofrecen un recurso prometedor como una alternativa a los aceites de pescado ${ }^{40}$. Las microalgas se utilizan en la actualidad en la producción controlada de ácidos grasos esenciales, libres de metales pesados y otro tipo de contaminantes ${ }^{41}$.

Si bien después del análisis de perfil de ácidos grasos presentó un contenido de DHA de $126 \mathrm{mg} / 200 \mathrm{~mL}$, que cubre el $63 \%$ de la recomendación de este ácido graso para mujeres gestantes y lactantes, en el análisis sensorial, la muestra con la mayor adición de aceite (M3 0,175\%) mostró diferencias significativas con la muestra M1 y M2, y fue la muestra de calidad general más baja por su reducido aroma frutal en la prueba de ordenamiento. A su vez, se observa que esta muestra tuvo una disminución del DHA, entre el día 2 y día 15 de almacenamiento $(20 \mathrm{mg} / 200 \mathrm{~mL}$ de yogur). Esto sugiere que las altas adiciones de aceite pueden afectar la vida útil del producto, específicamente en lo sensorial y a la estabilidad del ácido graso de interés. Ghorbanzade et al ${ }^{42}$ adicionó a un yogur nanoliposomas con aceite de pescado, los autores reportaron que a los 21 días de almacenamiento se observó una disminución del $50 \%$ en el contenido de DHA en el yogur al cual se le adicionó aceite de pescado encapsulado respecto al contenido inicial ${ }^{42}$ Contrario a lo anterior, Chávez-Servín et a ${ }^{43}$ identificaron la estabilidad del DHA en una formula láctea para infantes, por un periodo de 18 meses, y no identificaron degradación del DHA en ese tiempo En los resultados de este estudio, este hecho solo se observa con la concentración más alta del aceite de microalga.

Otros elementos que se observan en adiciones altas de aceite de microalga son las diferencias en lo incorporado, en comparación con lo identificado por perfil de ácidos grasos. En tal sentido, las muestras M2 y M3 mostraron no alcanzar el nivel esperado de adición de DHA, por la incorporación del aceite, teniendo diferencias de 10 mg y 14 mg menos para M2 y M3 respectivamente. No obstante, Robertson et al ${ }^{44}$, en el 2016, desarrollaron un yogur con adición de aceite de microalga Pavlova lutheri, en concentraciones de 0,2 y $0,5 \% \mathrm{p} / \mathrm{v}$, e identificaron que ninguna de las dos adiciones tuvo impacto significativo sobre la composición del yogur, la viscosidad, el pH, la viabilidad del cultivo iniciador o la separación del suero lácteo. Sin embargo, el análisis sensorial reveló que los yogures con la adición de aceite de microalga no fueron bien aceptados por los panelistas, contrario a los resultados obtenidos en el presente estudio, en los que los panelistas describieron características positivas de las muestras de yogur con adición de aceite, excepto para la muestra M3. En el análisis sensorial no se evidencian olores ni sabores rancios o que sugieran que en las muestras se percibía la sensación grasa de la adición del aceite, comparadas con el control. Estos son resultados contrarios a los resultados de Robertson et $\mathrm{a}^{44}$; sin embargo, las cantidades de aceite adicionadas en esta investigación fueron inferiores, lo que puede ser un factor determinante para la evaluación sensorial.
Se observa en los resultados que la relación n-6/n-3 disminuye a mayor adición de aceite. Esto era de esperarse, porque el DHA pertenece a la familia del AGPI n-3. Respecto al tema de salud, esta relación es importante, porque a valores más altos influye negativamente en la producción de eicosanoides con aumento en la producción de citoquinas proinflamatorias y puede afectar la fluidez de la membrana ${ }^{45}$. La relación de n-6/n-3 para Robertson et $a^{44}$ en sus muestras se presentó en la muestra control 2,2; relación mucho menor a la encontrada en el presente estudio, de 3,3 para M0, y en las que tenían adición del aceite de microalga 0,3 y 0,2 , más bajas también que en esta investigación (entre 0,8 y 0,4 ). Tal diferencia deriva de la mayor cantidad de aceite de microalga incorporado por ellos y a su vez de la leche usada para la producción del yogur.

La adición del aceite de microalga no afecta otros componentes como las proteínas (Tabla 2), esto es relevante porque el yogur y los productos lácteos son considerados, en el ámbito nutricional, un grupo de alimentos con un aporte adecuado de proteína de alto valor biológico, siendo importante en cualquier etapa del proceso vital humano por su aporte de macro y micronutrientes y sustancias bioactivas ${ }^{46}$. Si bien el diseño de este yogur se enfocó en el grupo de mujeres gestantes y lactantes, es clave sugerir que puede ser una opción para el consumo de DHA para cualquier tipo de población, teniendo en cuenta las recomendaciones individuales por grupos de edad o condición fisiológica. Incluso podría ayudar en el tratamiento de enfermedades crónicas como el hígado graso ${ }^{47,48}$. Se conoce que en las dietas actuales es difícil acceder a fuentes alimentarias con un buen aporte de DHA, por lo que este producto se convierte en un alimento promisorio.

Las ingestas dietéticas de DHA en mujeres embarazadas, en países como Australia ${ }^{49}$, Noruega ${ }^{50}$ y Canadá ${ }^{51}$, fueron de 150, 140 y 160 mg/día, respectivamente. En el estudio de Zhou ${ }^{52}$ en China, la ingesta promedio de DHA en las mujeres en gestación fue $20 \mathrm{mg} / \mathrm{d}$ áa, que está lejos de ser suficiente para satisfacer la demanda fetal. Vale la pena señalar que la ingesta dietética de DHA en mujeres embarazadas, en los países mencionados, no alcanza la recomendación de ingesta emitida por los consensos de $200 \mathrm{mg} / \mathrm{d}$ á. Se ha documentado que las mujeres chilenas durante el embarazo y la lactancia tienen una baja ingesta de alimentos que pueden ser fuente de ácidos grasos n-3, lo que se evidencia en una reducción de este ácido graso en eritrocitos y leche materna ${ }^{53}$. En otro estudio en gestantes chilenas, también se ratifica lo anterior y se menciona el desequilibrio en la ingesta de n-6/n-3, lo cual podría afectar negativamente el desarrollo fetal ${ }^{54}$. La anterior información evidencia la necesidad de aumentar el consumo de DHA en mujeres gestantes o lactantes, para lo cual, la adición en alimentos con ácidos grasos $\mathrm{n}-3$ puede ser una alternativa con el fin de atender la demanda adicional del feto, así como la fisiológica y las necesidades propias de las mujeres embarazadas o lactantes ${ }^{55}$. Se destaca que el yogur puede 
ser un alimento con alta viabilidad para vehiculizar la adición de n-3, porque es un producto que permanece a temperaturas bajas -refrigeración-, garantizando la estabilidad de los ácidos grasos; es consumido de manera frecuente en la población, hace parte de las guías alimentarias ${ }^{56}$, es tecnológicamente factible la adición de este aceite y, además, aporta micronutrientes importantes como calcio, fósforo y zinc ${ }^{57}$.

Los resultados de actividad antioxidante para las muestras de yogur se mostraron muy variables en este estudio (Figura 2). Se esperaría que el potencial antioxidante disminuyera durante el tiempo de almacenamiento por tratarse de un alimento adicionado con un componente inestable a la oxidación; no obstante, esto no fue evidente en los resultados de ABTS y fenoles totales. La misma composición del aceite de microalga, por estar estabilizado previamente con tocoferoles (vitamina E) y palmitato de ascorbilo (éster de vitamina $\mathrm{C}$ ), parece que tiene un potencial antioxidante que protege al aceite de su degradación y lo estabiliza en la incorporación en diferentes matrices alimentarias, como se evidencia en los resultados de esta investigación. También se han estudiado los factores importantes que contribuyen a la alta estabilidad oxidativa del yogur enriquecido con aceite de pescado, con especial énfasis en los posibles efectos antioxidantes de los péptidos liberados durante la fermentación del yogur. Farvin et $\mathrm{al}^{58}$ fraccionaron por ultrafiltración, usando membranas con tamaños de corte de $30 \mathrm{kDa}, 10 \mathrm{kDa}$ y $3 \mathrm{kDa}$, unas muestras de yogur enriquecido con aceite de pescado y evaluaron su actividad antioxidante. Ellos encontraron que las fracciones de menor peso molecular, 3-10 kDa y $<3 \mathrm{kDa}$, mostraron protección contra la oxidación del aceite de pescado en la misma medida que los caseinofosfopéptidos; también encontraron que el contenido de oxígeno del yogur también fue menor que el de la leche. Estos hallazgos sugieren que la mayor estabilidad oxidativa del yogur podría deberse a los péptidos que resultan ser antioxidantes y son liberados durante la fermentación de la leche por las bacterias del ácido láctico o por el menor contenido de oxígeno del yogur, que luego reduce el estrés oxidativo del aceite de pescado incorporado en el yogur; estos resultados fueron apoyados posteriormente por Najgebauer-Lejko et al ${ }^{59}$. A su vez, las propiedades antioxidantes de la leche fermentada son el resultado de la presencia de caseína, proteínas de suero, péptidos, aminoácidos, vitaminas $\mathrm{C}, \mathrm{E}, \mathrm{D}$ y $\mathrm{A}$, $\beta$-caroteno y sistemas enzimáticos (superóxido dismutasa, catalasa y glutatión peroxidasa), así como la presencia de ácido linoleico conjugado y la coenzima Q10, a lo que se le suma la actividad antioxidante de las bacterias ácido lácticas ${ }^{60}$. Estos datos pueden servir como explicación a la actividad y estabilidad antioxidante de las formulaciones de yogur realizadas en este estudio (Figura 2). El aumento final en los datos de ABTS puede ser producto de reacciones propias del proceso de fermentación, que puede llevar a la formación de péptidos $\mathrm{u}$ otras sustancias con potencial antioxidante y que su actividad puede aumentar durante el tiempo de almacenamiento, porque continúa los procesos de fermentación ${ }^{61}$.

Los resultados muestran altos niveles de inhibición del radical $\mathrm{ABTS}$, en casi todas las muestras en el transcurso de los días. Se observa que sobrepasa los $10.000(\mu \mathrm{mol}$ Trolox/L muestra) (Figura 2-A), lo cual es superior a lo reportado en el estudio de Ryckebosch ${ }^{62}$, quien estudió el potencial nutracéutico y antioxidante de la biomasa de diferentes microalgas para la extracción de n-3, con un resultado de 37 a $93 \mu \mathrm{M}$ Trolox/g de aceite de microalga. Es decir que la microalga por sí misma no proporciona actividad antioxidante alta, lo que resalta la adición que se realiza del tocoferol y palmitato de ascorbilo en el aceite usado en el presente estudio, siendo este último aditivo un antioxidante muy útil para evitar la oxidación en productos lácteos ${ }^{40,58}$. Lo anterior, a su vez, se evidencia en la prueba de ABTS, en la que la muestra control se comportó igual que las adicionadas con aceite $(>0,05)$, resultado no muy coherente, porque si fuera el aceite de microalga el que estuviera aportando la actividad antioxidante, se obtendrían diferencias significativas con la muestra control y los resultados no arrojan tales diferencias.

Los resultados de TBARS (Figura 2-C) se observan aumentados para el día 34, lo cual era de esperarse por el daño oxidativo que ocurre con los AGPI; sin embargo, no hay diferencias en el valor de TBARS en las muestras $(>0,05)$, es decir, la muestra control se oxida al mismo tiempo que las muestras adicionadas con el aceite. Este tiempo estimado con TBARS es superior al tiempo de almacenamiento que sugieren para este tipo de productos lácteos (20 días de vida útil) ${ }^{31}$.

Las limitaciones de este estudio fueron el desarrollo de solo dos lotes de yogur, lo que restringe el análisis estadístico de significancia en las pruebas que no se desarrollaron con triplicados o duplicados. Se tendría que complementar el análisis sensorial multidimensional, microbiológico y fisicoquímico para todas las muestras de yogur en varios periodos de tiempo, para determinar la vida útil del producto. Los resultados de actividad antioxidante se pudieron afectar por los aditivos que contiene el aceite de microalga; por lo tanto, estos resultados son poco concluyentes. En futuras investigaciones, sería importante someter el producto a una intervención con mujeres gestantes y lactantes, para comprobar su utilidad en esta población. A su vez, se tendría que evaluar la viabilidad técnica respecto al precio final del producto, debido a que el aceite de microalga tiene un costo superior al de otras fuentes de ácidos grasos n-3.

\section{CONCLUSIÓN}

Las bebidas lácteas fermentadas tipo yogur son matrices alimentarias adecuadas para incorporar ácidos grasos n-3, como el aceite de microalga, el cual tiene un elevado aporte de DHA y puede contribuir a las recomendaciones dietarias de este ácido graso en poblaciones que requieren un aporte especial de este componente en su dieta, como las mujeres gestantes y lactantes. Según los resultados, se 
sugiere que la incorporación máxima de aceite que cumple con las características de un producto de adecuada calidad global sea 0,125\%, para el aceite de microalga usado, el cual previamente está estabilizado con agentes antioxidantes, que lo protegen y reflejan la falla oxidativa posterior a los días recomendados de durabilidad de este tipo de productos lácteos. Sin embargo, hacen falta más estudios, para evaluar la efectividad del yogur en la población a la cual va dirigida.

\section{BIBLIOGRAFÍA}

1. Morales $P$ J, Valenzuela $B R$, González $M D$, González $E$ $M$, Tapia O G, Sanhueza C J, et al. New dietary sources of alpha-linolenic acid: a critical view. Rev Chil Nutr. 2012; 39: 79-87.

2. OMS. Marine oil supplementation to improve pregnancy outcomes [Internet]. 2011. Disponible en: https://www.who. int/elena/titles/bbc/fish_oil_pregnancy/en/.

3. Garg P, Pejaver RK, Sukhija M, Ahuja A. Role of DHA, ARA, \& phospholipids in brain development: An Indian perspective. Clin Epidemiol Glob Heal. 2017; 5: 155-162.

4. Valenzuela $B R$, Tapia $O G$, González $E M$, Valenzuela $B A$. Omega-3 fatty acids (EPA and DHA) and its application in diverse clinical situations. Rev Chil Nutr. 2011; 38: 356-367.

5. Rogers $L K$, Valentine CJ, Keim SA. DHA supplementation: Current implications in pregnancy and childhood. Pharmacol Res. 2013; 70: 13-19.

6. Gorjão R, Azevedo-Martins AK, Rodrigues HG, Abdulkader F, Arcisio-Miranda M, Procopio J, et al. Comparative effects of DHA and EPA on cell function. Pharmacol Ther. 2009; 122: 56-64.

7. Darwesh AM, Sosnowski DK, Lee TY, Keshavarz-Bahaghighat $H$, Seubert JM. Insights into the cardioprotective properties of $n-3$ PUFAs against ischemic heart disease via modulation of the innate immune system. Chem Biol Interact. 2019; 308: 20-44.

8. Echeverría $F$, Ortiz $M$, Valenzuela $R$, Videla $L A$. Long-chain polyunsaturated fatty acids regulation of PPARs, signaling: Relationship to tissue development and aging. Prostaglandins, Leukot Essent Fat Acids. 2016; 114: 28-34.

9. Martins DA, Custódio L, Barreira L, Pereira H, Ben-Hamadou $R$, Varela J, et al. Alternative sources of $n-3$ long-chain polyunsaturated fatty acids in marine microalgae. Mar Drugs. 2013; 11: 2259-2281.

10. Wang $X$, Wang $W X$. The three ' $B$ ' of fish mercury in China: Bioaccumulation, biodynamics and biotransformation. Environ Pollut. 2019; 250: 216-232.

11. Ruxton CHS, Reed SC, Simpson MJA, Millington KJ. The health benefits of omega-3 polyunsaturated fatty acids: a review of the evidence. I Hum Nutr Diet. 2004; 17: 449-459.

12. Bradbury J. Docosahexaenoic acid (DHA): An ancient nutrient for the modern human brain. Nutrients. 2011; 3: 529-554.

13. Gould JF, Treyvaud K, Yelland LN, Anderson PJ, Smithers LG, Gibson RA, et al. Does n-3 LCPUFA supplementation during pregnancy increase the IQ of children at school age? Follow-up of a randomised controlled trial. BMJ Open. 2016; 6: 11465-11474.

14. Luchtman DW, Song C. Cognitive enhancement by omega-3 fatty acids from child-hood to old age: Findings from animal and clinical studies. Neuropharmacology. 2013; 64: 550-565.

15. Moon K, Rao SC, Schulzke SM, Patole SK, Simmer K. Longchain polyunsaturated fatty acid supplementation in preterm infants. Cochrane Database Syst Rev. 2016; 12: 1-65
16. Gázquez A, Ruíz-Palacios M, Larqué E. DHA supplementation during pregnancy as phospholipids or TAG produces different placental uptake but similar fetal brain accretion in neonatal piglets. Br J Nutr. 2017; 118: 981-988.

17. FAO. Fats and fatty acids in human nutrition. Report of an expert consultation. FAO Food Nutr Pap. 2010; 91: 1-166.

18. Fleith M, Clandinin MT. Dietary PUFA for preterm and term infants: review of clinical studies. Crit Rev Food Sci Nutr. 2005; 45: 205-229.

19. Yurko-Mauro K, Kralovec J, Bailey-Hall E, Smeberg V, Stark JG, Salem N. Similar eicosapentaenoic acid and docosahexaenoic acid plasma levels achieved with fish oil or krill oil in a randomized double-blind four-week bioavailability study. Lipids Health Dis. 2015; 14: 1-9.

20. Lee EJ, Lee MW, No DS, Kim HJ, Oh SW, Kim Y, et al. Preparation of high purity docosahexaenoic acid from microalgae oil in a packed bed reactor via two-step lipase-catalysed esterification. J Funct Foods. 2016; 21: 330-337.

21. Fedorova-Dahms I, Marone PA, Bauter M, Ryan AS. Safety evaluation of DHA-rich Algal Oil from Schizochytrium sp. Food Chem Toxicol. 2011; 49: 3310-3318.

22. Adarme-Vega TC, Lim DKY, Timmins M, Vernen F, Li Y, Schenk PM. Microalgal biofactories: a promising approach towards sustainable omega-3 fatty acid production. Microb Cell Fact. 2012; 11: 96-106.

23. Koyande AK, Chew KW, Rambabu K, Tao Y, Chu D-T, Show P-L. Microalgae: A potential alternative to health supplementation for humans. Food Sci Hum Wellness. 2019; 8: 16-24.

24. Santos-Sánchez NF, Valadez-Blanco R, Hernández-Carlos B, Torres-Ariño A, Guadarrama-Mendoza PC, Salas-Coronado R. Lipids rich in $\omega-3$ polyunsaturated fatty acids from microalgae. Appl Microbiol Biotechnol. 2016; 100: 8667-8684.

25. Arterburn L., Boswell K., Koskelo E-K, Kassner S., Kelly C, Kyle D. A combined subchronic (90-day) toxicity and neurotoxicity study of a single-cell source of docosahexaenoic acid triglyceride (DHASCO ${ }^{\circledast}$ oil). Food Chem Toxicol. 2000; 38: 35-49.

26. AOAC. Fat in Milk Roese-Gottlieb Method. 905.02. 2002.

27. AOAC. Fatty acids in oils and fats AOAC 969.33. 2002.

28. AOAC. Fat (total, saturated, and unsaturated) in foods $A O A C$ 996.06. 2002.

29. Feng P, Weagant S, Grant M, Burkhardt W. BAM 4: Enumeration of Escherichia coli and the Coliform Bacteria (8. a ed). Food \& Drugs Administration: Microbiological Methods \& Bacteriological Analitical Manual (BAM) [En linea]. Maryland 2002.

30. ISO 6611/IDF 94: 2004. Milk and milk products: enumeration of colony-forming units of yeasts and/or moulds - Colonycount technique at 25 degrees C. ISO and IDF, Suiza, 2004.

31. FAO/OMS. Codex alimentarius: Milk and Milk Products [Internet]. 2011. Disponible en: http://www.fao.org/3/i2085e/ i2085e00.pdf

32. ISO. ISO 8587:2006/AMD 1:2013. sensory analysis. Methodology. Ranking. 2013.

33. ISO. ISO 11035:94. Sensory analysis. Identification and selection of descriptors for establishing a sensory profile by a multidimensional approach. 1994.

34. ISO. ISO 6658:2005. Sensory analysis. Methodology. General guidance. 2005.

35. AOAC 942,15. Acidity/Titratable Acidity. Maryland: AOAC Internaional, 1996.

36. AOAC 954,01: Protein/Crude Protein. Maryland: AOAC International, 2005. 
37. Re $R$, Pellegrini N, Proteggente $A$, Pannala A, Yang $M$, RiceEvans C. Antioxidant activity applying an improved ABTS radical cation decolorization assay. Free Radic Biol Med. 1999; 26: 1231-1237.

38. Sánchez N, Sepúlveda J, Rojano B. Development of a milk beverage with curuba (passiflora mollissima bailey) extracts as natural antioxidant. Rev Bio Agro. 2013; 11: 164-173.

39. Spolaore $P$, Joannis-Cassan C, Duran E, Isambert A. Commercial applications of microalgae. J Biosci Bioeng. 2006; 101: 87-96.

40. Valenzuela B A, Sanhueza C J, Valenzuela B R. Microalgae: A renewable source for obtaining omega-3 long-chain fatty acids for human and animal nutrition. Rev Chil Nutr. 2015; 42: 306-310.

41. Khozin-Goldberg I, Iskandarov U, Cohen Z. LC-PUFA from photosynthetic microalgae: Occurrence, biosynthesis, and prospects in biotechnology. Appl Microbiol Biotechnol. 2011; 91: 905-915.

42. Ghorbanzade T, Jafari SM, Akhavan S, Hadavi R. Nanoencapsulation of fish oil in nano-liposomes and its application in fortification of yogurt. Food Chem. 2017; 216: 146-152.

43. Chávez-Servín IL, Castellote Al, Martín M, Chifré R, Carmen López-Sabater M. Stability during storage of LC-PUFAsupplemented infant formula containing single cell oil or egg yolk. Food Chem. 2009; 113: 484-492.

44. Robertson RC, Gracia Mateo MR, O'Grady MN, Guihéneuf $F$, Stengel DB, Ross RP, et al. An assessment of the technofunctional and sensory properties of yoghurt fortified with a lipid extract from the microalga Pavlova lutheri. Innov Food Sci Emerg Technol. 2016; 37: 237-246.

45. Husted KS, Bouzinova $E$ V. The importance of $n-6 / n-3$ fatty acids ratio in the major depressive disorder. Medicina (Kaunas). 2016; 52: 139-147.

46. Verruck S, Balthazar CF, Ramon SR, Ramon S, Esmerino EA, Pimentel TC, et al. Dairy foods and positive impact on the consumer's health. Adv Food Nutr Res. 2019; 89: 95-164.

47. Hernandez-Rodas M, Valenzuela R, Videla L. Relevant Aspects of Nutritional and Dietary Interventions in Non-A/coholic Fatty Liver Disease. Int J Mol Sci. 2015; 16: 25168-25198.

48. Hernandez-Rodas MC, Morales P J, Valenzuela B R, Morales I $G$, Valenzuela B A. Benefits of $n-3$ long-chain polyunsaturated fatty acids in non-alcoholic fatty liver disease. Rev Chil Nutr. 2016; 43: 196-205.

49. Parker G, McClure G, Hegarty BD, Smith IG. The validity of a food frequency questionnaire as a measure of PUFA status in pregnancy. BMC Pregnancy Childbirth. 2015; 15: 60-65.
50. Brantsæter $A L$, Haugen $M$, Thomassen $Y$, Ellingsen DG, Ydersbond TA, Hagve T-A, et al. Exploration of biomarkers for total fish intake in pregnant Norwegian women. Public Health Nutr. 2010; 13: 54-62.

51. Innis SM, Elias SL. Intakes of essential n-6 and n-3 polyunsaturated fatty acids among pregnant Canadian women. Am J Clin Nutr. 2003; 77: 473-478.

52. Zhou YB, Li HT, Trasande L, Wang LL, Zhang YL, Si KY, et al. A correlation study of DHA intake estimated by a FFQ and concentrations in plasma and erythrocytes in mid- and late pregnancy. Nutrients. 2017; 9: 1256-1269.

53. Barrera C, Valenzuela R, Chamorro R, Bascuñán K, Sandoval I, Sabag N, et al. The Impact of Maternal Diet during Pregnancy and Lactation on the Fatty Acid Composition of Erythrocytes and Breast Milk of Chilean Women. Nutrients. 2018; 10: 839-853.

54. Bascuñán K, Valenzuela R, Chamorro R, Valencia A, Barrera C, Puigrredon $C$, et al. Polyunsaturated Fatty Acid Composition of Maternal Diet and Erythrocyte Phospholipid Status in Chilean Pregnant Women. Nutrients. 2014; 6: 4918-4934.

55. Makrides M, Gibson RA. Long-chain polyunsaturated fatty acid requirements during pregnancy and lactation. Am J Clin Nutr. 2000; 71: 307-311.

56. FAO. Food-based dietary guidelines. 2018. Disponible en: http://www.fao.org/nutrition/education/food-dietary-guidelines/ regions/latin-america-caribbean/en/.

57. USDA. USDA Food Composition Databases. 2019. Disponible en: https://fdc.nal.usda.gov/.

58. Schmitt D, Tran N, Peach J, Bauter M, Marone P. Toxicologic evaluation of DHA-rich algal oil: Genotoxicity, acute and subchronic toxicity in rats. Food Chem Toxicol. 2012; 50: 3567-3576.

59. Najgebauer-Lejko D, Sady M. Estimation of the antioxidant activity of the commercially available fermented milks. Acta Sci Pol Technol Aliment. 2015; 14:387-396.

60. Fardet $A$, Rock $E$. In vitro and in vivo antioxidant potential of milks, yoghurts, fermented milks and cheeses: a narrative review of evidence. Nutr Res Rev. 2018; 31: 52-70.

61. Rahmawati IS, Suntornsuk W. Effects of Fermentation and Storage on Bioactive Activities in Milks and Yoghurts. Procedia Chem. 2016; 18: 53-62.

62. Ryckebosch E, Bruneel C, Termote-Verhalle R, Goiris K, Muylaert K, Foubert I. Nutritional evaluation of microalgae oils rich in omega-3 long chain polyunsaturated fatty acids as an alternative for fish oil. Food Chem. 2014; 160: 393-400. 